\title{
Consequências à criança do uso de drogas durante a gestação: um artigo de revisão
}

\section{Consequences of illicit drug use during pregnancy to the child: review article}

\author{
Raquel da Silveira Kataoka de Paulaํ. \\ 1 Residente de Pediatria do Hospital Universitário Walter Cantídio (HUWC), Universidade Federal do Ceará (UFC), Fortaleza, \\ Ceará, Brasil.

\section{RESUMO}

O crescimento do uso de drogas ilícitas entre as mulheres em idade fértil é preocupante. O uso de drogas durante a gestação é causa de morbidades que podem se apresentar no feto, no neonato ou em qualquer etapa do desenvolvimento infantil. Esta revisão reuniu informações sobre prevalência, métodos de rastreio e consequências clínicas da exposição fetal à cocaína, metanfetamina, maconha e opioides. As taxas de exposição podem variar de 2 a $40 \%$ entre os estudos. Nos métodos de rastreio, a análise de cabelo materno se mostrou a mais sensível, a análise de mecônio é de mais fácil execução e os questionários aplicados às mães têm baixa sensibilidade. Várias consequências para a gestação e para a criança são relacionadas à exposição a drogas. Entre elas encontramos maior incidência de perdas gestacionais, prematuridade, baixo peso ao nascer, Apgar $<7$ no quinto minuto, internações em UTI e óbito neonatal. Síndrome de abstinência neonatal, anomalias congênitas, déficit de atenção, problemas de comportamento, prejuízos no desenvolvimento motor, cognitivo e de linguagem são os acometimentos mais encontrados na infância. Concluímos serem necessárias políticas de prevenção, identificação das crianças expostas e seu acompanhamento durante toda a infância.

Palavras-chave: Drogas ilícitas. Gestação. Criança. Recém-nascido.

\section{ABSTRACT}

The growth of illicit drugs use by childbearing age women is worrying. The use of drugs during pregnancy causes morbidities that may occur in the developing fetus, the neonate, or in any stage of child development. This review gathered information on prevalence, screening methods, and clinical consequences of fetal exposure to cocaine, methamphetamine, marijuana, and opioids. Exposure rates can range from 2 to $40 \%$ on the studies. In the screening methods, maternal hair analysis was the most sensible test, meconium analysis is the easiest to perform and the questionnaires applied to mothers have low sensibility. Several consequences to the gestation and to the child are associated to drug exposure. Among them, we found a higher incidence of miscarriages, prematurity, low birth weight, 5-minute Apgar score $<7$, intensive care unit admissions and neonatal death. Neonatal abstinence syndrome, congenital anomalies, attention deficit disorder, behavioral problems, and motor, cognitive and language impairments are the most frequent consequences in childhood. We conclude that prevention policies, the identification of exposed children, and monitoring them throughout childhood are necessary.

Keywords: Street drugs. Pregnancy. Child. Infant, newborn.

Autor correspondente: Raquel da Silveira Kataoka de Paula, Rua Florêncio Coelho Holanda, 600, Maraponga, Fortaleza, Ceará. CEP: $60711-220$. Telefone: +55 85 98696-0350. E-mail: raquel_kataoka@yahoo.com.br

Conflito de interesses: Não há qualquer conflito de interesses por parte de qualquer um dos autores.

Recebido em: 12 Dez 2016; Revisado em: 01 Abr 2017; Aceito em: 01 Abr 2017. 


\section{INTRODUÇÃO}

O uso de drogas tem se mostrado um grave problema de saúde. A ONU, em relatório divulgado em 2012, estima que 5\% de toda a população adulta mundial tenha utilizado algum tipo de droga ilícita durante o ano de 2010. Embora globalmente esse índice tenha se mantido estável, em alguns países tem crescido. Foi observado que esse consumo tem sido mais importante entre os jovens e tem aumentado nos países em desenvolvimento. De forma semelhante, foi observado que, embora os homens permaneçam como grupo mais atingido, a prevalência entre as mulheres tem se elevado, principalmente na faixa etária jovem. Estima-se que, nos países em desenvolvimento, esse padrão de crescimento será mantido. ${ }^{1}$

Embora não haja dados recentes quanto ao uso de drogas no Brasil, especialistas perceberam um crescimento no uso de cocaína em 2010. ${ }^{1}$ Dados de 2001 do Observatório Brasileiro de Informações sobre Drogas mostram que 19,4\% dos entrevistados já havia utilizado algum tipo de droga ilícita e que esse número subiu para $22,8 \%$ em $2005 .^{2}$

O Centro de Monitoramento Europeu mostra que a prevalência do abuso de drogas entre adultos jovens, incluindo mulheres em idade fértil, tem crescido na Europa nas últimas duas décadas. ${ }^{3}$ Nesse grupo etário, as taxas de consumo na Espanha são as maiores daquele continente. Entre as mulheres de 15 a 34 anos de idade, $13,2 \%, 3,2 \%$ e 1,4\% admitiram o consumo de maconha, cocaína e ecstasy, respectivamente, nos últimos 12 meses. ${ }^{4}$ É observado também que o consumo é cada vez mais precoce. ${ }^{5}$

Esse crescimento no consumo de drogas entre as mulheres em idade fértil traz preocupação. Há relatos de que aproximadamente $90 \%$ das usuárias de drogas nos Estados Unidos estejam em idade fértil. ${ }^{6}$ Um levantamento americano mostrou que 5,5\% das mães relataram uso de drogas na gravidez. Há que se pontuar que este dado provavelmente é subestimado, devido ao estigma associado à questão abordada. ${ }^{7}$ Outros estudos referentes à população americana revelam taxas de exposição gestacional que variam entre $2 \% \mathrm{e}$ $40 \% .{ }^{8,9}$ Um estudo brasileiro detectou presença de cocaína ou maconha em $6 \%$ das pacientes gestantes estudadas. ${ }^{10}$

Dessa forma, a exposição gestacional é um fato importante por ser relativamente frequente e pela morbidade perinatal que ocasiona. ${ }^{5}$ Aumenta os riscos de complicações obstétricas e tem graves consequências para o desenvolvimento fetal e para a criança, tanto no período neonatal, quanto em etapas posteriores da vida. Além disso, traz custos econômicos e sociais. $^{7}$

Mais de 75\% das crianças expostas a drogas têm graves problemas de saúde. Quando analisadas crianças não expostas a esse risco, a porcentagem de crianças com afecções cai para $27 \%$. O custo para tratamento das crianças expostas é duas vezes maior que para as crianças não expostas.?

No que tange às complicações obstétricas, há relatos de aborto, parto prematuro, insuficiência placentária, descolamento prematuro de placenta, óbito intrauterino e aumento da incidência de doenças infecciosas e sexualmente transmissíveis. . $^{5,9,11,12}$

Os agravos para as crianças expostas incluem baixo peso ao nascimento, aumento na incidência de reanimação neonatal, hemorragias intracranianas, outras alterações diagnosticadas no período neonatal (malformações congênitas, distúrbios ácido-base, síndrome de abstinência, distúrbios motores) e alterações observadas durante todo o desenvolvimento infantil até a idade adulta (déficits no desenvolvimento motor e cognitivo, transtornos comportamentais, aumento nos índices de abuso de drogas).

Diante da gravidade do problema em questão, a identificação e o seguimento das gestantes usuárias de drogas mostramse necessários. A abordagem a ser realizada é um assunto controverso, havendo quem defenda até mesmo a reclusão para tratamento compulsório e a classificação da situação como negligência ou abuso infantil. ${ }^{6,7}$ Diante disso, vemos a necessidade de mais estudos.

O tratamento de gestantes usuárias deveria ser uma prioridade para os governos. O esclarecimento da população para esse tema nas esferas médica, legal e social poderia reduzir barreiras para o tratamento, além de ajudar as pacientes a reduzir os riscos das complicações associadas. ${ }^{7,12}$

O acompanhamento médico adequado das crianças expostas a drogas no período gestacional também é de extrema importância. Essas crianças estão sujeitas a um grande número de agravos que podem apresentar-se nas diversas etapas do desenvolvimento e, por isso, necessitam de seguimento contínuo. Somente o acompanhamento adequado permitirá a prevenção de complicações e o diagnóstico precoce, permitindo um tratamento otimizado e reduzindo prejuízos para os pacientes e para a sociedade.

\section{METODOLOGIA}

Revisão não sistemática na literatura nacional e internacional, utilizando o banco de dados MEDLINE/PUBMED e órgãos para monitorização do uso de drogas do Brasil, Europa e Organização das Nações Unidas (ONU). Foram selecionados os artigos que abordassem o uso de drogas de abuso durante a gestação, seu rastreio e consequências para o feto e para a criança nas diversas fases do desenvolvimento. Foram pesquisados os termos "drogas de abuso", "gestação", "criança" e "recém-nascido".

\section{IDENTIFICAÇÃO DE EXPOSIÇÃO GESTACIONAL A DROGAS}

Diante da informação de que muitas gestantes não relatam o uso de drogas durante o atendimento hospitalar e de que muitos neonatos não apresentam sintomatologia chamativa, surgiu a necessidade de um método laboratorial que detectasse o abuso de drogas durante a gestação. ${ }^{5,13}$ Várias estratégias foram 
utilizadas para determinar de forma objetiva quais neonatos haviam sido expostos a drogas ilícitas, possibilitando seu seguimento adequado.

Os testes de sangue e urina, que são os mais usualmente utilizados para detecção de drogas, são de pouca utilidade nessa situação, por refletirem apenas uso recente. As análises em urina utilizadas como triagem são capazes de detectar o uso apenas no período de 1 a 4 dias que precedem a coleta das amostras. ${ }^{7,13,14}$ Em busca de testes que permitissem a identificação dos neonatos expostos a drogas de abuso em qualquer período da gestação, foram realizados estudos com análise de mecônio, cabelo materno e cabelo do recémnascido.

Os diferentes estudos confirmaram a existência de uma importante disparidade entre as informações obtidas por questionários aplicados às mães e as informações obtidas em análises objetivas, demonstrando a existência de uma subdeclaração do uso pelas gestantes. . $^{45,13}$

Em estudo realizado por Garcia-Algar et al., entre outubro de 2002 e fevereiro de 2004, foi encontrada exposição a drogas de abuso em 10,9\% das pacientes estudadas, através da determinação de metabólitos em amostra de mecônio, enquanto a entrevista materna mostrava uma prevalência de $2,9 \%$. De $57(4,7 \%)$ casos positivos para heroína, apenas 4 $(0,3 \%)$ haviam declarado o consumo em questionário. $\mathrm{O}$ consumo de cocaína foi relatado em menos de 50\% dos casos identificados por análise. A análise de mecônio sempre confirmou o consumo declarado. ${ }^{16}$

García-Serra et al. encontraram, em seu estudo de 2010, uma prevalência de $15,9 \%$ para uso de drogas na gestação em análise de cabelo materno e mecônio. No mesmo estudo, a prevalência encontrada por questionário foi de $1,9 \%{ }^{4}$ De forma análoga, Bessa et al. encontraram em estudo brasileiro de análise de cabelo materno uma prevalência de $4 \%$ para uso apenas de maconha, 1,7\% para uso apenas de cocaína e 3\% para uso de ambas. Nenhuma das pacientes havia relatado o uso de drogas durante a entrevista. ${ }^{17}$

Em estudo de Lester et al., no grupo de neonatos com mecônio positivo para cocaína e opioides, $38 \%$ das mães haviam negado o uso de drogas. ${ }^{8}$

A análise de mecônio tem sido o método mais utilizado e suas principais vantagens são a facilidade de obtenção da amostra e a boa aceitação pelos pais. Tem como desvantagens o período para coleta de menos de $72 \mathrm{~h}$, o custo mais elevado e a impossibilidade de detectar exposições no primeiro trimestre da gestação, já que a formação do mecônio tem início entre a $12^{\mathrm{a}}$ e a $16^{\mathrm{a}}$ semanas de gestação. A análise do cabelo materno permite ampliar a janela de detecção e estimar em que período houve a exposição, considerando o crescimento de $1 \mathrm{~cm}$ ao mês. Como desvantagem, fornece estimativa direta da exposição materna e estimativa apenas indireta de exposição fetal, além de apresentar resultados falso-positivos, provavelmente por exposição passiva. A análise do cabelo fetal também se mostrou útil para a detecção da exposição.
O cabelo fetal inicia seu crescimento por volta da vigésima semana de gestação e sua taxa de crescimento não é conhecida, fornecendo uma janela de detecção menor que os outros métodos. ${ }^{4,15,13,14,18,19}$

Em estudo comparativo entre os dois métodos mais utilizados (análise de mecônio e análise de cabelo materno), concluiuse que a análise de cabelo materno parece ser mais sensível que a análise em mecônio, principalmente para maconha. García-Serra et al. em seu estudo observaram que, dos 11 casos positivos para maconha, apenas 3 foram confirmados em mecônio e, dos 7 casos positivos para cocaína, 6 foram confirmados em mecônio. ${ }^{4}$ Em estudo de análise em cabelo materno, Friguls et al. não encontraram casos positivos em entrevista que resultassem negativos por análise. ${ }^{15}$ As análises de cabelo materno também mostraram-se mais sensíveis para detecção de cocaína e opiáceos que o mecônio, embora tenham apresentado resultados falso-positivos. ${ }^{19}$ Outros estudos relataram amostras negativas em mecônio e em cabelo fetal, havendo a mãe relatado o uso de metanfetamina durante a gestação. ${ }^{18,20}$

Pela passagem transplacentária variável, a análise de mecônio tem se mostrado mais útil para avaliar a exposição fetal do que para a identificação do uso materno. Estudos têm demonstrado que o método mais sensível para detecção do uso de drogas durante a gestação seria a análise de cabelo materno. Porém, pela sua alta sensibilidade e pela facilidade de coleta de amostras, a análise de mecônio tem sido apontada como o método mais adequado de rastreio. ${ }^{15,19}$ Nesse contexto, em locais de alta prevalência, há recomendações de aconselhamento preventivo específico e de implementação de rastreio gestacional, além do neonatal. ${ }^{15}$

O rastreio e monitoramento da exposição gestacional ainda gera discussões, principalmente pelos aspectos legais, e necessita de mais estudos. $\mathrm{O}$ rastreio adequado possibilita uma análise mais acurada dos efeitos no feto. Ao se aumentar o conhecimento sobre os efeitos fisiológicos, bioquímicos e comportamentais da exposição gestacional a drogas, poder-seiam oferecer soluções para uma melhor prevenção, tratamento e redução no número de crianças expostas. ${ }^{7}$

\section{CONSEQUÊNCIAS ÀS CRIANÇAS}

Em estudos para avaliar a prevalência da exposição gestacional a drogas, encontra-se um número de abortos prévios mais elevado entre as mães usuárias. Além disso, também são relatados menor peso e comprimento ao nascer para os neonatos expostos, maior frequência de Apgar $<7$ no quinto minuto, maior frequência de internação em UTI e óbito neonatal, sem que houvesse diferença étnica ou social entre os grupos de mães expostas e não expostas., ${ }^{91}, 15,16$

Martín Mardomingo et al. encontraram em seu estudo que $79 \%$ dos neonatos expostos a drogas apresentaram síndrome de abstinência neonatal, com uma idade média de $26 \mathrm{~h}$. Nesse estudo, não foram utilizados métodos analíticos para identificação dos casos, foram considerados expostos os casos em que havia relato materno ou sintomatologia fetal. ${ }^{5}$ No 
mesmo estudo, $34 \%$ dos neonatos possuíam problema familiar importante e foram encaminhados a centros de acolhimento por determinação judicial.

Muitos recém-nascidos expostos a drogas não apresentam sintomatologia chamativa durante o período hospitalar, mas devem ser acompanhados por haver relatos de prejuízos observados em diversas idades. Em estudo de Friguls et al., foram identificadas exposições a maconha, cocaína e metanfetamina, sem que houvesse relatos de síndrome de abstinência e sem diferenças significativas quanto ao Apgar, eventos hipoglicêmicos e distúrbios ácido-básicos. ${ }^{15}$

Existem poucos estudos que avaliam alterações morfológicas na placenta que possam justificar os comprometimentos obstétricos e fetais. ${ }^{9}$ Foi demonstrado que ela permite a passagem da maioria das drogas utilizadas pela mãe., ${ }^{6,21}$ Seus metabólitos podem acumular na placenta ou no líquido amniótico e induzir alterações deciduais (endoteliais, angiogênicas e de síntese hormonal). Em estudo publicado em 2012, foi observado que o uso de drogas induz alterações na vascularização placentária que poderiam levar a baixa perfusão, justificando os efeitos observados nos neonatos (acidose e baixos peso e comprimento). ${ }^{9}$

Estudos de neuroimagem por ressonância magnética têm sido realizados para avaliar a existência de alterações do sistema nervoso central relacionadas à exposição gestacional a drogas. Tais estudos têm demonstrado alterações de córtex pré-frontal e gânglios da base que sugerem a existência de neurotoxicidade específica, mas possuem alto custo e são realizados em amostras pequenas, havendo necessidade de estudos similares comprobatórios. A maioria dos estudos é transversal, prejudicando as informações do passado e havendo possibilidade de que os pacientes do estudo tenham sofrido efeito das drogas em períodos diferentes do período gestacional. O conhecimento das alterações estruturais e funcionais no sistema nervoso central pode predizer comprometimentos neurocomportamentais futuros, além de melhorar o diagnóstico e o tratamento das crianças e adolescentes acometidos. ${ }^{22}$

A maioria das mulheres que utilizam drogas durante a gestação expõe-se a mais de uma substância, tornando difícil a análise dos impactos de cada droga individualmente. Os fatores de confusão também incluem os elementos sociais envolvidos, como pobreza e cuidados familiares deficientes, que podem estar envolvidos no modelamento cerebral.

\section{Cocaína}

A exposição pré-natal à cocaína tem sido associada à prematuridade, baixo peso ao nascimento, microcefalia, anomalias congênitas cardiovasculares e urogenitais, deficiências neurocomportamentais neonatais em estado de alerta e autorregulação, prejuízo no desenvolvimento motor, risco de acidente cerebrovascular intraútero e síndrome de abstinência. ${ }^{9,15,23-25}$ Foram também relatados prejuízos a longo prazo, incluindo déficits de inteligência, linguagem, funções executivas e controle de impulso e atenção. ${ }^{21}$ Alguns estudos não têm encontrado evidência que sustente a associação, indicando que pode haver correlação com a exposição a álcool, tabaco e maconha..$^{20,25} \mathrm{Em}$ estudos mais recentes, porém, encontra-se relação entre o uso de cocaína e baixo peso ao nascer, baixo comprimento e menor perímetro cefálico, excluindo outros parâmetros que poderiam afetar as medidas antropométricas. ${ }^{10,15}$

Existem três explicações possíveis para o impacto da cocaína no desenvolvimento cerebral. A primeira envolve os efeitos diretos da cocaína em neurotransmissores. Receptores de monoamina exercem papel trófico no desenvolvimento cerebral, que pode ser prejudicado pelo excesso de monoamina secundário aos efeitos da cocaína. Há também interação com outros neurotransmissores, incluindo GABA e glutamato. A segunda explicação envolve os efeitos vasoconstrictores que levariam a alterações placentárias e, posteriormente, hipóxia fetal. A terceira envolve alterações de expressão gênica que alteram as funções neuroendócrinas fetais..$^{9,21,23}$

Chris Derauf et al. em seu artigo de revisão relatam os achados de neuroimagem por ressonância magnética em pacientes expostos à cocaína no período gestacional, incluindo infartos corticais, esquizencefalia, paquigiria e aumento da incidência de hemorragias periventriculares, cistos subependimários e periventriculares. ${ }^{21}$

Há evidências de redução da área do corpo caloso proporcional ao grau de exposição, redução das substâncias cinzentas em lobo parietal direito (cujo volume foi relacionado a habilidades sensório motoras, acuidade visual e a escore de construções sintáticas aos 6 anos de idade) e em lobo occipital esquerdo (cujo volume está relacionado à acuidade visual neonatal, memória visual aos 12 meses e performance visual motora aos 6 anos). ${ }^{26}$

Há relatos de redução de área de caudado e putâmen que podem estar relacionados ao déficit de atenção. Redução no tálamo pode justificar déficits de aprendizagem. ${ }^{21,27}$ Utilizando testes com crianças entre 8 e 9 anos, Sheinkopf et al. observaram hipersinal em regiões frontal e estriada, enquanto as crianças não expostas apresentavam ativação de córtex occipital e giro fusiforme. Esse achado sugere diferença funcional em controle cognitivo e atenção. ${ }^{28}$ Em adolescentes, há relato de redução de $10 \%$ no fluxo sanguíneo cerebral. ${ }^{29}$

Os estudos que demonstraram redução no caudado e na substância cinzenta cortical global por cocaína não excluíram outros tóxicos e esse achado pode refletir outras exposições ou associações de drogas. ${ }^{30,31}$

\section{Metanfetamina}

Exposição prenatal à metanfetamina induz menor peso ao nascimento, menor perímetro cefálico, prematuridade e alterações neurocomportamentais. Estudos têm mostrado efeitos adversos nas esferas física, emocional, cognitiva e social, com aumento dos índices de déficit de atenção, déficit de aprendizagem e agressão. Porém, pode haver fator de confusão com outras drogas. ${ }^{13,21,32}$ 
Estudo inglês de 2012 submeteu crianças de 12 meses de idade, cujas mães relataram uso de metanfetamina associada ou não a outras drogas durante a gestação, a testes para desenvolvimento motor, cognitivo e de linguagem. As crianças expostas a altas doses apresentaram baixo desenvolvimento motor e cognitivo, mesmo após correções para exposição a outras drogas. ${ }^{33}$

Estudo americano de 2012 avaliou crianças de 3 e 5 anos de idade expostas a metanfetamina durante a gravidez na busca por problemas de comportamento. Em ambas as idades, foram encontradas reações emocionais exageradas e problemas de ansiedade e depressão. Aos 5 anos, a exposição mostrou relação com a prevalência de déficit de atenção e hiperatividade. Nesse estudo foram feitas correções para o uso de outras drogas. ${ }^{34}$

Estudos com ratos observaram remodelamento sináptico em terminais axônicos e efeitos neurotóxicos serotoninérgicos. ${ }^{35,36}$ Estudos em animais e adultos evidenciaram efeitos neurotóxicos no córtex pré-frontal, nucleus accumbens, putâmen, caudado, globus pallidus, hipocampo, giro cingular e amigdala. Tais regiões são importantes para o desenvolvimento, controle emocional, atenção, linguagem e cognição. Todas essas funções já foram relacionadas como possivelmente atingidas pelo uso materno de drogas. Acredita-se que a metanfetamina possa exercer seus efeitos deletérios por efeito neurotóxico direto, vasoconstrição e anorexia materna..$^{21}$

Em estudo de neuroimagem por ressonância magnética, foi observada redução volumétrica significativa em diversas áreas subcorticais (globus pallidus, putâmen e hipocampo). Essas alterações foram relacionadas a prejuízos em atenção sustentada e a atrasos em memória verbal. ${ }^{37}$

Apesar dos estudos possuírem fatores de confusão como exposição conjunta a outras drogas, amostra pequena, fatores médicos e sociais, os achados sugerem que as estruturas subcorticais citadas e o circuito pré-frontal estriado envolvidos em atenção e memória podem ser prejudicados pelos efeitos da metanfetamina no cérebro em desenvolvimento. ${ }^{38-40}$

\section{Maconha}

A maconha é a droga ilícita mais utilizada durante a gestação. $\mathrm{O}$ uso de maconha durante a gestação é relacionado a déficit de atenção, impulsividade e déficit de aprendizado, memória, raciocínio verbal e visão abstrata. Hátambém relatos de aumento dos índices de delinquência. ${ }^{10,21}$ Moraes et al encontraram alterações em performance neurocomportamental (estado de alerta, autorregulação e excitabilidade) entre neonatos de termo com exposição pré-natal a maconha. ${ }^{41}$ Também foram descritas taxas mais elevadas de distúrbio ácido-básico. ${ }^{9}$ Em crianças entre 9 e 12 anos de idade expostas a altas doses de maconha, foram observados menores perímetros cefálicos. ${ }^{21}$

Os mecanismos envolvidos incluem efeitos diretos e indiretos sobre os neurotransmissores dopaminérgicos no nucleus accumbens. $\mathrm{O}$ efeito indireto é mediado pela ativação dos receptores canabinoides $\mathrm{CB} 1$, causando a liberação de opioides endógenos que vão atuar liberando dopamina no nucleus accumbens. $^{42}$ Esses mecanismos têm sido relacionados a interferência em sinaptogênese e em proliferação, migração e diferenciação neuronais. ${ }^{43}$

Os estudos de ressonância magnética evidenciaram redução global no volume da substância cinzenta, porém sem a exclusão do uso de outras drogas. Mostraram também que a redução na substância branca é maior nos pacientes expostos a maconha e cocaína que nos pacientes expostos somente à cocaína. Também documentaram, durante a realização de testes, aumento das atividades no córtex pré-frontal e no córtex pré-motor direito e redução na atividade cerebelar esquerda, podendo indicar necessidade de maior esforço para conclusão dos testes propostos. ${ }^{21}$

Shabani et al. encontraram alterações significativas na descendência de ratas expostas a maconha. As alterações foram observadas nas propriedades eletrofisiológicas intrínsecas dos neurônios cerebelares de Purkinje e estavam associadas a mudanças severas nas performances motoras. Os neurônios de Purkinje exibiam amplitudes maiores nos picos de potenciais de ação e maiores períodos de latência. ${ }^{44}$

\section{Opioides}

A exposição aos opioides tem sido associada à síndrome de abstinência neonatal, além de complicações obstétricas. ${ }^{9}$ Existem poucos estudos que analisam os efeitos dos opioides no desenvolvimento infantil e apenas um estudo de neuroimagem para investigar alterações em sistema nervoso central. $^{21}$

\section{PAPEL DA OCITOCINA NA NEUROADAPTAÇÃO}

Pesquisas nas últimas quatro décadas têm demonstrado que a ocitocina participa em formas de neuroadaptação, incluindo aprendizado e memória. ${ }^{45}$ Há evidências de que ela tenha papel no comportamento reprodutivo e na modulação da plasticidade comportamental, como no aprendizado associativo. Ela influencia o comportamento e a memória sociais e tem sido demonstrada sua participação no comportamento materno. ${ }^{46,47}$ Sua concentração aumenta durante a gestação e, no período pós-parto, atua modulando os comportamentos ansiosos e depressivos. $^{48}$

No estudo do papel da ocitocina na drogadição, a dependência tem sido avaliada como "aprendizado patológico" e a tolerância desenvolvida a algumas drogas também é avaliada como uma espécie de aprendizado. É provável que a ocitocina atue, via inibição, em alguns processos neuroadaptativos envolvendo aprendizado e memória. ${ }^{45}$ Experimento realizado por Qi e col. mostrou que tratamento com ocitocina reduziu os efeitos estimulantes da metanfetamina. ${ }^{49}$ Sarnyai e Kovacs demonstraram que administração aguda de cocaína aumenta a concentração de ocitocina no hipocampo, enquanto a administração crônica a reduz..$^{50}$

A exposição gestacional à cocaína tem efeitos dinâmicos nos níveis de ocitocina e seus receptores. Seus efeitos na 
mãe prejudicam o cuidado materno, ao reduzir os níveis de ocitocina, impactando o ambiente em que a criança está inserida e trazendo consequências para o desenvolvimento infantil. Para o feto em formação, traz prejuízos para o comportamento social e aumenta a agressividade na idade adulta. $^{48}$

Nelson et al. realizaram estudo com roedores expostos a cocaína e encontrou evidências de que a cocaína prejudique a interação entre mãe e filho no período pós-parto, aumentando a negligência infantil. Esse efeito era dependente da dose utilizada, período e duração da exposição. ${ }^{51}$ Os estudos com outras drogas são raros, mas alguns têm encontrado evidências de que o álcool e o tabaco possuam efeito semelhante ao da cocaína na redução dos níveis de ocitocina. ${ }^{48}$ As mães usuárias de cocaína frequentemente possuem desordens do humor, fazem uso de álcool e tabaco e pertencem a grupo socioeconômico mais baixo. Todos esses fatores causam impacto nos cuidados maternos e, no grupo de crianças expostas a drogas na gestação, o índice de abandono chega a ser 20 vezes maior que na população geral. ${ }^{52}$

Os efeitos da exposição gestacional à cocaína no feto em formação podem perdurar por longos períodos. Várias alterações que já foram relacionadas a essa exposição (como resposta de estresse, comportamento opositor e chances elevadas de abuso de drogas) têm se mostrado moduladas pela ocitocina. $^{48}$

As crianças expostas demonstram menos reações negativas quando separadas das mães e durante o trabalho Still-Face, que observa reações da criança à ausência de interação materna. ${ }^{53}$ Também se mostram mais difíceis de acalmar e são mais irritadas, enquanto suas mães demonstram resposta exagerada à irritabilidade..$^{54}$

Em estudos conduzidos com roedores, foram observadas alterações comportamentais que se mantiveram a longo prazo.

\section{REFERÊNCIAS}

1. United Nations Office on Drugs and Crime. World Drug Report 2012 [Internet]. New York: UNODC; 2012 june [acesso $17 \mathrm{fev}$ 2015]. Disponível em: https://www.unodc.org/unodc/en/data-andanalysis/WDR-2012.html

2. Carlini EA, Galduróz JC, Noto AR, Nappo SA. I Levantamento Domiciliar sobre o Uso de Drogas Psicotrópicas no Brasil: Estudo Envolvendo as 107 Maiores Cidades do País - 2001 [Internet]. São Paulo: CEBRID, UNIFESP; 2002. [acesso em 17 fev 2015]. Disponível em: http:// abramd.org/wp-content/uploads/2014/06/I_Levantamento_Domiciliar_ sobre_o_Uso_de_Drogas_Psicotr\% $\%$ C3\%B3picas_no_Brasil.pdf

3. European Monitoring Centre for Drugs and Drug Addiction. European Monitoring Centre for Drugs and Drug Addiction Statistical bulletin 2011 [Internet]. Lisbon: EMCDDA; 2011. [acesso 17 fev 2015]. Disponivel em: http://www.emcdda.europa.eu/stats11

4. García-Serra J, Ramis J, Simó S, Joya X, Pichini S, Vall O, et al. Matrices biológicas alternativas para detectar la exposición prenatal a drogas de abuso en el tercer trimestre de la gestación. An Pediatr (Barc). 2012;77(5):323-8.
Os adolescentes expostos à cocaína no período gestacional podem apresentar agressividade, menos interação social e comportamento ansioso. Depressão também é observada na idade adulta, além do comportamento ansioso e agressivo. Os roedores que passaram por exposição gestacional apresentaram alterações nos níveis de ocitocina no período neonatal e experimentaram alterações comportamentais, mas não apresentavam alterações nos níveis de ocitocina na idade adulta. Os roedores que foram criados por mãe exposta a cocaína possuem alterações nos níveis de ocitocina por maiores períodos. ${ }^{48}$

\section{CONCLUSÕES}

A exposição gestacional a drogas é um importante problema de saúde pública. Nesta revisão, demonstrou-se sua alta prevalência, embora ainda subdiagnosticada, e a importância do rastreio para a identificação dos casos. Sabendo que muitos neonatos se apresentam assintomáticos durante a internação hospitalar e que os questionários aplicados às mães são pouco sensíveis na identificação do uso de drogas, existe a necessidade da implementação de métodos analíticos para esse fim.

Conhecendo os inúmeros agravos à saúde a que estão sujeitas as crianças expostas a drogas no período gestacional, seu acompanhamento deve receber especial atenção. $\mathrm{O}$ seguimento multidisciplinar adequado a longo prazo permite prevenção, diagnóstico precoce e tratamento dos transtornos neuropsicomotores e comportamentais.

Devido às graves consequências à criança, além do alto custo relacionado à morbidade e do alto custo social, o combate ao uso de drogas durante a gravidez deve ser uma prioridade. Dessa forma, o tratamento das gestantes usuárias de drogas é essencial, embora ainda controverso pelos aspectos legais, e necessita de mais estudos. O esclarecimento da população quanto aos riscos envolvidos deve ser reforçado.

5. Mardomingoa MA, Sáncheza GS, Guerrerob SM, Quesadaa CC, Méndeza CP, Péreza JL. Consumo de drogas durante el embarazo y morbilidad neonatal: cambios epidemiológicos en los últimos 10 años. An Pediatr (Barc). 2003;58(6):574-9.

6. Kuczkowski KM. The effects of drug abuse on pregnancy. Curr Opin Obstet Gynecol. 2007;19(6):578-85.

7. Huestis MA, Choo RE. Drug abuse's smallest victims: in utero drug exposure. Forensic Sci Int. 2002;128(1-2):20-30.

8. Lester BM, ElSohly M, Wright LL, Smeriglio VL, Verter J, Bauer $\mathrm{CR}$, et al. The Maternal Lifestyle Study: drug use by meconium toxicology and maternal self-report. Pediatrics. 2001;107(2):309-17.

9. Ortigosa S, Friguls B, Joya X, Martinez S, Mariñoso ML, Alameda F, et al. Feto-placental morphological effects of prenatal exposure to drugs of abuse. Reprod Toxicol. 2012;34(1):73-9.

10. Bessa MA, Mitsuhiro SS, Chalem E, Barros MM, Guinsburg R, Laranjeira R. Underreporting of use of cocaine and marijuana during 
the third trimester of gestation among pregnant adolescents. Addict Behav. 2010;35(3):266-9.

11. Bauer CR, Shankaran S, Bada HS, Lester B, Wright LL, KrauseSteinrauf H, et al. The Maternal Lifestyle Study: drug exposure during pregnancy and short-term maternal outcomes. Am J Obstet Gynecol. 2002;186(3):487-95.

12. Kashanian M, Baradaran HR, Hatami H. The effect on pregnancy outcome of drug (substance) abuse during pregnancy. Eur Psychiatry. 2013;28(suppl. 1):1.

13. Wada M, Sugimoto Y, Ikeda R, Isono K, Kuroda N, Nakashima K. Determination of methamphetamine in neonatal hair and meconium samples: estimation of fetal drug abuse during pregnancy. Forensic Toxicol. 2012;30(1):80-3.

14. Wada M, Ikeda R, Kuroda N, Nakashima K. Analytical methods for abused drugs in hair and their applications. Anal Bioanal Chem. 2010;397(3):1039-67.

15. Friguls B, Joya X, Garcia-Serra J, Gómez-Culebras M, Pichini S, Martinez S. Assessment of exposure to drugs of abuse during pregnancy by hair analysis in a Mediterranean island. Addiction. 2012;107(8):1471-9.

15. García-Algar O, Vall Combelles O, Puig Sola C, Mur Sierra A, Scaravelli G, Pacifici R, et al. Exposición prenatal a drogas de abuso a través del análisis de meconio en una población de bajo nivel socioeconómico en Barcelona. An Pediatr (Barc). 2009;70(2):151-8.

17. Garcia-Bournissen F, Rokach B, Karaskov T, Koren G. Methamphetamine detection in maternal and neonatal hair: implications for fetal safety. Arch Dis Child Fetal Neonatal Ed. 2007;92(5):F351-5.

18. Ostrea EM, Knapp DK, Tannenbaum L, Ostrea AR, Romero A, Salari V, et al. Estimates of illicit drug use during pregnancy by maternal interview, hair analysis, and meconium analysis. J Pediatr. 2010;138(3):344-8.

19. Gray TR, Kelly T, LaGasse LL, Smith LM, Derauf C, Grant $\mathrm{P}$, et al. New meconium biomarkers of prenatal methamphetamine exposure increase identification of affected neonates. Clin Chem. 2010;56(5):856-60.

20. Ellis JE, Byrd LD, Sexson WR, Patterson-Barnett CA. In utero exposure to cocaine: a review. Southern Medical Journal. 1993;86(7):725-31.

21. Derauf C, Kekatpure M, Neyzi N, Lester B, Kosofsky B. Neuroimaging of children following prenatal drug exposure C. Semin Cell Dev Biol. 2009;20(4):441-54.

22. Chasnoff IJ, Griffith DR. Cocaine: clinical studies of pregnancy and the newborn. Ann N Y Acad Sci. 1989;562:260-6.

23. Lester B, Padbury JF. The third pathophysiology of prenatal cocaine exposure. Dev Neurosci. 2009;3(1-2):23-35.

24. Shankaran S, Lester BM, Das A, Bauer CR, Bada HS, Lagasse $\mathrm{L}$, et al. Impact of maternal substance use during pregnancy on childhood outcome. Semin Fetal Neonatal Med 2007;12(2):143-50.

25. Frank DA, Augustyn M, Knight WG, Pell T, Zuckerman

B. Growth, Development, and Behavior in Early Childhood
Following Prenatal Cocaine Exposure A Systematic Review. JAMA. 2001;285(12):1613-25.

26. Dow-Edwards DL, Benveniste H, Behnke M, Bandstra ES, Singer LT, Hurd YL, et al. Neuroimaging of prenatal drug exposure. Neurotoxicol Teratol. 2006;28(3):386-402.

27. Gabriel M, Taylor C. Prenatal exposure to cocaine impairs neuronal coding of attention and discriminative learning. Ann N Y Acad Sci 1998;846:194-212.

28. Sheinkopf SJ, Lester BM, Sanes JN, Eliassen JC, Hutchison E, Seifer R, et al. Functional MRI and response inhibition in children exposed to cocaine in utero: Preliminary findings. Dev Neurosci. 2009;31(1-2):159-66.

29. Rao H, Wang J, Giannetta J, Korczykowski M, Shera D, Avants $\mathrm{BB}$, et al. Altered resting cerebral blood flow in adolescents with in utero cocaine exposure revealed by perfusion functional MRI. Pediatrics. 2007;120(5):e1245-54.

30. Avants BB, Hurt H, Giannetta JM, Epstein CL, Shera DM, Rao $\mathrm{H}$, et al. Effects of heavy in utero cocaine exposure onadolescent caudatemorphology. Pediatr Neurol. 2007;37(4):275-9.

31. Rivkin MJ, Davis PE, Lemaster JL, Cabral HJ, Warfield SK, Mulkern RV, et al. Volumetric MRI study of brain in children with intrauterine exposure to cocaine, alcohol, tobacco, and marijuana. Pediatrics. 2008;121(4):741-50.

32. Smith LM, Lagasse LL, Derauf C, Grant, P, Shah R, Arria A, et al. Prenatal methamphetamine use and neonatal neurobehavioral outcome. Neurotoxicol Teratol. 2008;30(1):20-8.

33. Singer LT, Moore DG, Min MO, Goodwin J, Turner JJ, Fulton S, et al. One-Year Outcomes of Prenatal Exposure to MDMA and Other Recreational Drugs. Pediatrics. 2012;130(3):407-13.

34. LaGasse LL, Derauf C, Smith LM, Newman E, Shah R, Neal C, et al. Prenatal Methamphetamine Exposure and Childhood Behavior Problems at 3 and 5 Years of Age. Pediatrics. 2012;129(4):681-8.

35. Weissman AD, Caldecott-Hazard S. Developmental neurotoxicity to methamphetamines. Clin Exp Pharmacol Physiol. 1995;22(5):372-4.

36. Jeng W, Wong AW, Ting AKR, Wells PG. Methamphetamineenhanced embryonic oxidative DNA damage and neurodevelopmental deficits. Free Radic Biol Med. 2005;39(3):317-26.

37. Chang L, Smith LM, LoPresti C, Yonekura ML, Kuo J, Walot I, et al. Smaller subcortical volumes and cognitive deficits in children with prenatal methamphetamine exposure. Psychiatry Res. 2004;132(2):95-106.

38. Teicher MH, Anderson CM, Polcari A, Glod CA, Maas LC, Renshaw PF. Functional deficits in basal ganglia of children with attention-deficit/hyperactivity disorder shown with functional magnetic resonance imaging relaxometry. Nat Med. 2000;6(4):470-3.

39. Castellanos FX, Giedd JN, Marsh WL, Hamburger SD, Vaituzis AC, Dickstein DP, et al. Quantitative brain magnetic resonance imaging in attention-deficit hyperactivity disorder. Arch Gen Psychiatry. 1996;53(7):607-16.

40. Castellanos FX, Sharp WS, Gottesman RF, Greenstein DK, Giedd $\mathrm{JN}$, Rapoport JL. Anatomic brain abnormalities in monozygotic 
twins discordant for attention deficit hyperactivity disorder. Am J Psychiatry. 2003;160(9):1693-6.

41. Barros MC, Guinsburg R, Peres CA, Mitsuhiro S, Chalem E, Laranjeira RR. Exposure to marijuana during pregnancy alters neurobehavior in the early neonatal period. J Pediatr. 2006;149(6):781-7.

42. Tanda G, Goldberg SR. Cannabinoids: reward, dependence, and underlying neurochemical mechanisms - a review of recent preclinical data. Psychopharmacology (Berl). 2003;169(2):115-34.

43. Harkany T, Guzman M, Galve-Roperh I, Berghuis P, Devi LA, Mackie $\mathrm{K}$. The emerging functions of endocannabinoid signaling during CNS development. Trends Pharmacol Sci. 2007;28(2):83-92.

44. Shabani M, Hosseinmardi N, Haghani M, Shaibani V, Janahmadia M. Maternal exposure to the CB1 cannabinoid agonist WIN 55212-2 produces robust changes in motor function and intrinsic electrophysiological properties of cerebelar purkinje neurons in rat offspring. Neuroscience. 2011;172(2011):139-52.

45. Sarnyai Z, Kovács GL. Oxytocin in learning and addiction: From early discoveries to the present. Pharmacol Biochem Behav. 2014;119:3-9.

46. Beets I, Janssen T, Meelkop E, Temmerman L, Suetens N, Rademakers S, et al. Vasopressin/oxytocin-related signaling regulates gustatory associative learning in C. elegans. Science. 2012;338(6106):543-5.
47. Garrison JL, Macosko EZ, Bernstein S, Pokala N, Albrecht DR, Bargmann CI. Oxytocin/vasopressin-related peptides have an ancient role in reproductive behavior. Science. 2012;338(6106):540-3.

48. Williams SK, Johns JM. Prenatal and gestational cocaine exposure: Effects on the oxytocin system and social behavior with implications for addiction. Pharmacol Biochem Behav. 2014;119:10-21.

49. Qi J, Yang JY, Song M, Li Y, Wang F, Wu CF. Inhibition by oxytocin of methamphetamineinduced hyperactivity related to dopamine turnover in the mesolimbic region in mice. Naunyn Schmiedebergs Arch Pharmacol. 2008;376(6):441-8.

50. Sarnyai Z, Kovács GL. Role of oxytocin in the neuroadaptation to drugs of abuse. Psychoneuroendocrinology. 1994;19(1):85-117.

51. Johns JM, Nelson CJ, Meter KE, Lubin DA, Couch CD, Ayers A, et al. Dose-dependent effects of multiple acute cocaine injections on maternal behavior and aggression in Sprague-Dawley rats. Dev Neurosci. 1998;20(6):525-32.

52. Eiden RD, Foote A, Schuetze P. Maternal cocaine use and caregiving status: group diferences in caregiver and infant risk variables. Addict Behav. 2007;32(3):465-76.

53. Lewis MW, Phillips G, Bowser M, DeLuca S, Johnson HL, Rosen TS. Cocaine-exposed infant behavior during Still-Face: risk factor analyses. Am J Orthopsychiatry. 2009;79(1):60-70.

54. Schuetze P, Molnar DS, Eiden RD. Profiles of reactivity in cocaine-exposed children. J Appl Dev Psychol. 2012;33(6):282-93.

\section{Como citar:}

Paula RS. Consequências à criança do uso de drogas durante a gestação: um artigo de revisão. Rev Med UFC. 2018 jan-mar;58(1):45-52. 